\title{
Activation of AKT by hypoxia: a potential target for hypoxic tumors of the head and neck
}

\author{
Hanneke Stegeman ${ }^{*}$, Johannes H Kaanders ${ }^{1}$, Deric L Wheeler ${ }^{2}$, Albert J van der Kogel ${ }^{1}$, Marieke M Verheijen ${ }^{1}$,
} Stijn J Waaijer ${ }^{1}$, Mari lida², Reidar Grénman³, Paul N Span ${ }^{1}$ and Johan Bussink

\begin{abstract}
Background: Only a minority of cancer patients benefits from the combination of EGFR-inhibition and radiotherapy in head and neck squamous cell carcinoma (HNSCC). A potential resistance mechanism is activation of EGFR and/or downstream pathways by stimuli in the microenvironment. The aim of this study was to find molecular targets induced by the microenvironment by determining the in vitro and in vivo expression of proteins of the EGFR-signaling network in 6 HNSCC lines. As hypoxia is an important microenvironmental parameter associated with poor outcome in solid tumors after radiotherapy, we investigated the relationship with hypoxia in vitro and in vivo.
\end{abstract}

Methods: Six human HNSCC cell lines were both cultured as cell lines (in vitro) and grown as xenograft tumors (in vivo). Expression levels were determined via western blot analysis and localization of markers was assessed via immunofluorescent staining. To determine the effect of hypoxia and pAKT-inhibition on cell survival, cells were incubated at $0.5 \% \mathrm{O}_{2}$ and treated with MK-2206.

Results: We observed strong in vitro-in vivo correlations for EGFR, pEGFR and HER2 ( $\left.r_{s}=0.77, p=0.10, r_{s}=0.89, p=0.03\right)$ and $r_{s}=0.93, p=0.02$, respectively), but not for pAKT, pERK1/2 or pSTAT3 (all $r_{s}<0.55$ and $p>0.30$ ). In vivo, pAKT expression was present in hypoxic cells and pAKT and hypoxia were significantly correlated $\left(r_{s}=0.51, p=0.04\right)$. We confirmed in vitro that hypoxia induces activation of AKT. Further, pAKT-inhibition via MK-2206 caused a significant decrease in survival in hypoxic cells ( $p<0.01$ ), but not in normoxic cells.

Conclusions: These data suggest that (p)EGFR and HER2 expression is mostly determined by intrinsic features of the tumor cell, while the activation of downstream kinases is highly influenced by the tumor microenvironment. We show that hypoxia induces activation of AKT both in vitro and in vivo, and that hypoxic cells can be specifically targeted by PAKT-inhibition. Targeting PAKT is thus a potential way to overcome therapy resistance induced by hypoxia and improve patient outcome.

Keywords: Head and neck cancer, Tumor microenvironment, Hypoxia, pAKT, EGFR

\section{Background}

Head and neck squamous cell carcinoma (HNSCC) is the sixth most common cancer with 500.000 new diagnoses per year worldwide [1]. Recent research findings have resulted in a better understanding of the biologic features of HNSCC tumors, which has led to the development of new therapeutic agents targeting specific molecules important for tumor growth and cell survival.

\footnotetext{
* Correspondence: h.stegeman@rther.umcn.nl

'Department of Radiation Oncology, Radboud University Nijmegen Medical Centre, PO Box 9101, 6500 HB, Nijmegen, The Netherlands

Full list of author information is available at the end of the article
}

One of these new successful targeting agents is Cetuximab, a monoclonal antibody against the Epidermal Growth Factor Receptor (EGFR), which improves survival in HNSCC patients treated with radiotherapy [2]. However, despite this success, a significant proportion of patients does not benefit from the addition of antiEGFR treatment. In addition, most clinical trials find no correlation between EGFR expression assessed by immunohistochemistry and response to treatment with EGFR inhibitors [3].

To improve patient outcome with these new intensified treatments, a large amount of research has been

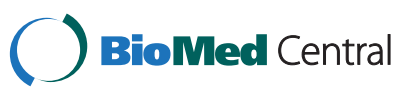


focused on identifying resistance mechanisms using different in vitro models [4-7]. However, it is uncertain to what extent these in vitro results can be translated towards the in vivo situation. A potential reason for the absence of a correlation between EGFR expression and response to EGFR inhibition is the EGFRindependent activation of signaling pathways, including the phosphatidylinositol-3-kinase (PI3-K)/protein kinase $B$ (AKT) pathway, by other stimuli in the microenvironment [8]. The tumor microenvironment is complex and includes fluctuating oxygen and nutrient gradients, which are not present in standard 2D cell culture assays, but which can have a great impact on tumor behavior and treatment response. Hypoxia is an important microenvironmental parameter known to induce the transcription and activation of a wide variety of proteins and is an inherent negative factor for treatment outcome in solid tumors, including treatment outcome after radiotherapy [9-11]. Hence, it is of great importance to discover molecular targets that could be used to specifically kill hypoxic cells and consequently improve patient outcome.

Therefore, the aim of this study was to find molecular targets in the EGFR-pathway, which are induced by the microenvironment and thus not detected in standard in vitro assays. For this, we determined the in vitro and in vivo expression of proteins involved in the EGFRsignaling network in HNSCC lines both cultured as cell lines and grown as xenograft tumors. We investigated the tyrosine kinase receptors EGFR and human epidermal growth factor receptor 2 (HER2), and the activated form of kinases of the main signaling pathways downstream of EGFR: protein kinase B (AKT), extracellular signalregulated kinase 1/2 (ERK1/2), and signal transducer and activator of transcription 3 (STAT3) [12]. Using this method, we were able to determine that pAKT, pERK1/2 and PSTAT3 were differentially expressed between cells in culture and in tumors, as an indication that these proteins were likely to be influenced by the tumor microenvironment. More importantly, we observed that AKT is activated by hypoxia both in vivo and in vitro and that the hypoxic cells are more sensitive to AKT-inhibition. These data implicate that pAKT-inhibition could be a promising way to specifically target hypoxic tumors in the clinic and improve outcome after a variety of treatments, including radiotherapy and EGFR-inhibition.

\section{Methods}

\section{Cell lines}

Six human head and neck squamous cell carcinoma cell lines (UT-SCC lines) were both cultured in vitro and grown as xenografts in nude mice. The characteristics of the cell lines are shown in Table 1. Cells were cultured in T75 culture flasks, under humidified conditions
Table 1 Characteristics of UT-SCC cell lines

\begin{tabular}{lllll}
\hline Cell line & TNM $^{*}$ & Primary tumor location & Type of lesion & Grade \\
\hline UT-SCC5 & $\mathrm{T}_{1} \mathrm{~N}_{1} \mathrm{M}_{0}$ & Tongue & Primary & 2 \\
UT-SCC8 & $\mathrm{T}_{2} \mathrm{~N}_{0} \mathrm{M}_{0}$ & Supraglottic larynx & Primary & 1 \\
UT-SCC15 & $\mathrm{T}_{1} \mathrm{~N}_{0} \mathrm{M}_{0}$ & Tongue & Recurrence & 1 \\
UT-SCC29 & $\mathrm{T}_{2} \mathrm{~N}_{0} \mathrm{M}_{0}$ & Glottic larynx & Primary & 1 \\
UT-SCC38 & $\mathrm{T}_{2} \mathrm{~N}_{0} \mathrm{M}_{0}$ & Glottic larynx & Primary & 2 \\
UT-SCC45 & $\mathrm{T}_{3} \mathrm{~N}_{1} \mathrm{M}_{0}$ & Floor of mouth & Primary & 3 \\
\hline
\end{tabular}

*TNM status of primary tumors according to the International Union against Cancer (1997).

Note: Grade: 1, well differentiated; 2 , moderately differentiated; 3 , poorly differentiated.

$\left(37^{\circ} \mathrm{C}, 5 \% \mathrm{CO}_{2}\right)$, and passaged weekly or twice weekly in DMEM containing $2 \mathrm{mM} \mathrm{L}$-glutamine, $1 \%$ nonessential amino acids, $20 \mathrm{mM}$ Hepes, 10 units $/ \mathrm{ml}$ penicillin, 10 units $/ \mathrm{ml}$ streptomycin, and $10 \%$ fetal bovine serum.

\section{Hypoxic incubation and MK-2206 treatment}

To determine expression levels after hypoxia and/or AKTinhibition, UT-SCC5 and UT-SCC15 cells were treated overnight (16h) with 0 or $2 \mu \mathrm{M}$ MK-2206 (Selleckchem, Houston, TX, USA) under standard normoxic conditions and thereafter incubated under normoxic conditions or under hypoxic conditions $\left(0.5 \% \mathrm{O}_{2}, \mathrm{H} 35\right.$ hypoxystation, Don Whitley Scientific Ltd., West Yorkshire, UK) for $1 \mathrm{~h}$.

To assess cell survival after hypoxia and/or AKTinhibition, UT-SCC5 and UT-SCC15 cells were seeded in 96-well plates. After the cells were allowed to attach overnight under standard normoxic conditions, 0 or $2 \mu \mathrm{M}$ MK-2206 was added and cells were incubated under normoxic conditions or under hypoxic conditions $\left(0.5 \% \mathrm{O}_{2}\right)$ for $72 \mathrm{~h}$. Cell survival was determined $72 \mathrm{~h}$ after normoxic or hypoxic incubation using a CellCounting Kit-8 assay (CCK8, Sigma-Aldrich Chemie BV, Zwijndrecht, The Netherlands).

\section{Xenograft tumors}

Animal experiments were performed using 6-8 week-old $\mathrm{BALB} / \mathrm{c}$ nu/nu mice. Of all six carcinoma cell lines, $5 \times 10^{6}$ cells were injected subcutaneously into the flank. Tumor size was measured by the same technician twice a week. Tumors with a diameter of $4 \mathrm{~mm}$ or larger were harvested or passaged. For passaging, the tumor was excised and cut into $1 \mathrm{~mm}^{3}$ tumor pieces. The tumor pieces were then subcutaneously implanted into the flank. In this study first, second, and third passage tumors were analyzed. One hour before tumor excision, animals were injected intraperitonally with $0.5 \mathrm{ml}$ of saline containing $2 \mathrm{mg}$ pimonidazole hydrochloride (1-[(2-hydroxy-3-piperidinyl) propyl]-2-nitroimidazole hydrochloride, Natural Pharmaceuticals International Inc., Research Triangle Park, NC, USA) to label hypoxic cells. After excision, tumors were immediately frozen in liquid nitrogen. The number of 
harvested tumors ranged from 2 to 4 per cell line with a total of 19 .

Animals were kept in a specific pathogen-free unit in accordance with institutional guidelines. All experiments were approved by the Animal Experiments Committee of the Radboud University Nijmegen Medical Centre.

\section{Western blot analysis}

To determine protein expression in vitro and in vivo, cultured cells or tumor sections were lysed, cell debris was removed by centrifugation and protein was quantitated using a standard Bradford absorbance assay. Proteins (25 $\mu \mathrm{g}$ per lane) were separated by SDS-PAGE and blotted onto PVDF membrane. Membranes were incubated with the appropriate primary antibodies followed by incubation with HRP-conjugated antibodies. Finally, proteins were detected with an ECL chemiluminescence system. Antibodies against the following antigens were used: EGFR, pEGFR (Y1173), HER2, AKT, STAT3, and HRPconjugated goat-anti-rabbit IgG, goat-anti-mouse IgG and donkey-anti-goat IgG were purchased from Santa Cruz Biotechnology Inc. (Santa Cruz, CA, USA). pHER2 (Y1221/1222), pAKT(S473), pERK1/2(T202/Y204), ERK1/ 2 , and pSTAT3(Y705) were purchased from Cell Signaling Technology (Beverly, MA, USA) and $\alpha$-tubulin was obtained from Calbiochem (San Diego, CA, USA).

To obtain a quantitative measure for total protein expression, the integrated optical density (IOD) of the chemiluminescent signal was measured using ImageJ software (NIH, Bethesda, MD, USA). IOD values of all proteins were normalized to those of $\alpha$-tubulin.

\section{Immunohistochemical staining, image acquisition and analysis of tumor sections}

To determine localization of protein expression in vivo, frozen tumor sections $(5 \mu \mathrm{m})$ were thawed, fixed in acetone $\left(4^{\circ} \mathrm{C}\right)$ and rehydrated in PBS. Two consecutive tumor sections from each tumor were stained for $\mathrm{pAKT}$ or pimonidazole in combination with EGFR and vessels. EGFR and pAKT were stained with the same antibodies used for western blot analysis. The antibody against pimonidazole was a gift from J.A. Raleigh (University of North Carolina) and 9F1 supernatant, a rat monoclonal antibody against mouse endothelium, was a gift from the Department of Pathology, Radboud University Nijmegen Medical Centre. EGFR was detected by incubation with Cy3-conjugated donkey-anti-goat IgG (Jackson Immuno Research Laboratories Inc., West Grove, PA, USA), pAKT and pimonidazole by incubation with Alexa488-conjugated donkey-anti-rabbit IgG (Molecular Probes, Leiden, The Netherlands), and 9F1 by incubation with Alexa647-conjugated chicken-anti-rat IgG (Molecular Probes). Stained sections were mounted in Fluorostab (ICN Pharmaceuticals, Inc, Zoetermeer, The Netherlands).

Stained tumor sections were scanned on a digital image processing system consisting of a 12-bit chargecouple device camera (Micromax, Roper Scientific Inc., Trenton, NJ, USA) on a fluorescence microscope (Axioskop, Zeiss Göttingen, Germany) and a computercontrolled motorized stepping stage, using IPLab software. Each section was sequentially scanned three times at $100 x$ magnification, yielding an image of hypoxia (pimonidazole) or pAKT-expression, an image of EGFRexpression and an image of the vasculature structures (9F1). One pseudo-colored composite image was reconstructed from the individual microscope images. Using this composite image, total tumor area was delineated and non-tumor tissue, necrotic area and staining artifacts were excluded from the analysis. Thereafter, thresholds for the fluorescence signals were interactively set at intensities where the steepest gradient occurred between background and foreground intensity levels, and grey value images were converted to binary images. Using ImageJ software (NIH, Bethesda, MD, USA), the fraction positive for $\mathrm{PAKT}$ or hypoxia was calculated by dividing the tumor area positive for the respective marker by the total tumor area. The overlap fraction of EGFR and pAKT was calculated by dividing the tumor area positive for both markers by the tumor area positive for EGFR.

\section{Statistics}

Statistical analyses were performed using Prism 4.0c (GraphPad Software, Inc., LA Jolla, CA, USA). Correlations between parameters were assessed using the Spearman correlation test. The significance of differences in cell survival between different treatments was assessed using a Kruskal-Wallis test in combination with a Dunn's multiple comparison test. P-values $<0.05$ were considered to be significant.

\section{Results}

Expression of activated kinases, but not of tyrosine kinase receptors, induced by tumor microenvironment

Using western blot analyses, expression levels of various proteins involved in the EGFR-signaling network were assessed in 6 different HNSCC cell lines both grown in culture (in vitro) and grown as xenograft tumors (in vivo). As example, western blots for (p)EGFR and pAKT are shown in Figure 1 (western blot images of (p)HER2, (p)ERK1/2 and (p)STAT3 are shown in Additional file 1: Figure S1).

The tyrosine kinase receptors EGFR and HER2 showed a wide variation of expression levels in both cells and tumors. Overexpression of these membrane receptors seems to be an intrinsic feature of the cell lines as for both EGFR and HER2 a strong correlation was found between in vitro and in vivo expression levels $\left(\mathrm{r}_{\mathrm{s}}=0.77\right.$, 


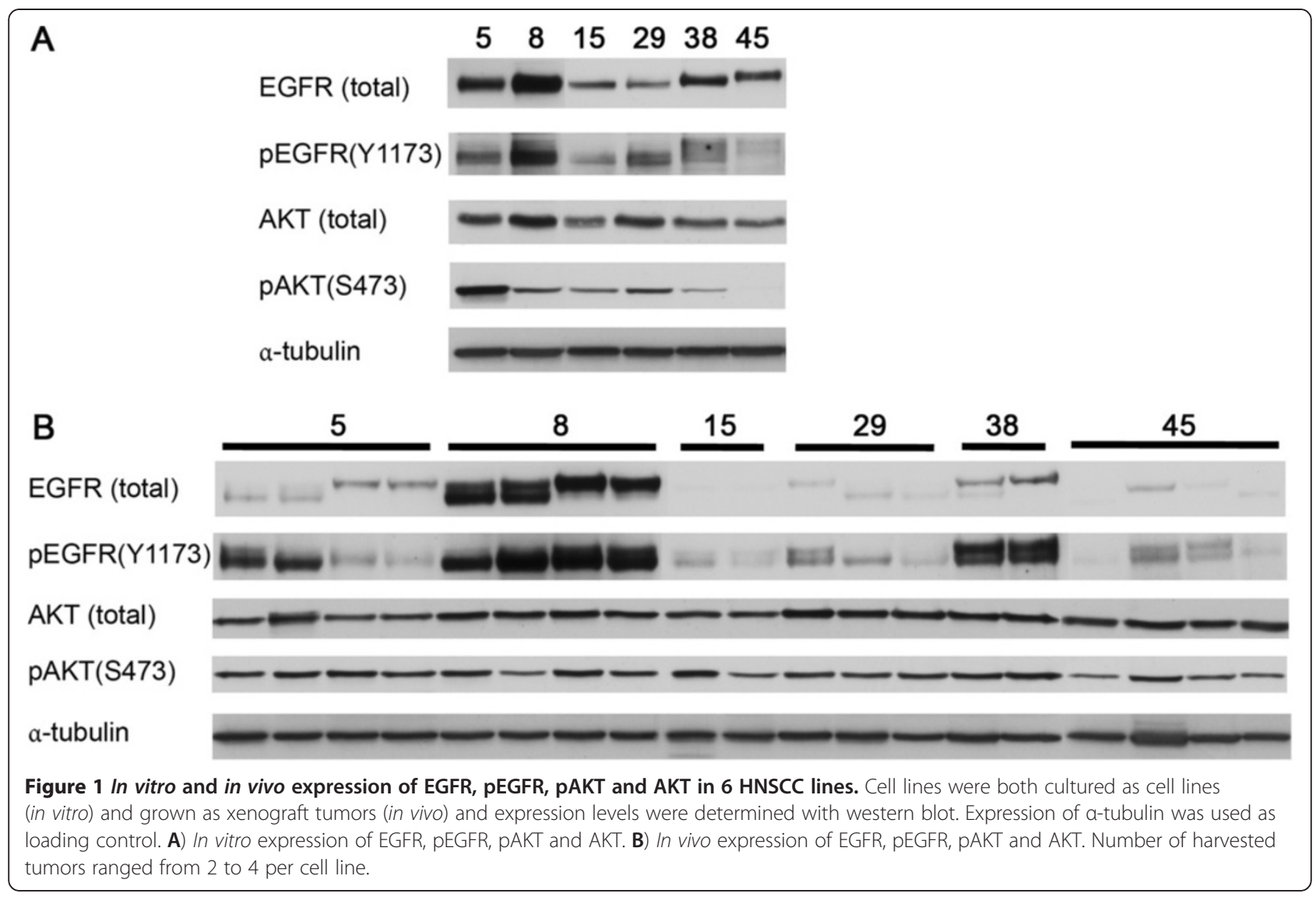

$\mathrm{p}=0.10$ and $\mathrm{r}_{\mathrm{s}}=0.93, \mathrm{p}=0.02$, respectively) (Figure 2A). Although there was only a trend towards a correlation between in vitro and in vivo EGFR expression $(\mathrm{p}=0.10)$, the correlation for the activated form pEGFR was significant $\left(\mathrm{r}_{\mathrm{s}}=0.89, \mathrm{p}=0.03\right)$. This was not the case for $\mathrm{pHER} 2$ $\left(\mathrm{r}_{\mathrm{s}}=0.26, \mathrm{p}=0.66\right)$, which showed different levels of expression in the tumors, but was invariably low in vitro.

In contrast to the tyrosine kinase receptors, no in vitro-in vivo correlation was observed for the activated kinases pAKT, pERK1/2 or pSTAT3 (all $\mathrm{r}_{\mathrm{s}}<0.55$ and $\mathrm{p}>0.30$ ) (Figure $2 \mathrm{~B}$ ). The lack of correlation was predominantly due to the fact that there was a wide range of expression levels in vitro while in vivo the kinases were activated at relatively high levels in all tumor lines (Figure 1 and 2B). These results suggest that stimuli in the tumor microenvironment activate the different cell signaling pathways and consequently lead to relatively high in vivo levels of pAKT, pERK1/2 and pSTAT3.

\section{pAKT expression present in hypoxic cells in vivo}

A variety of stimuli within the tumor microenvironment can activate kinases like AKT, ERK1/2 and STAT3, including hypoxia. Therefore, EGFR, pAKT, and hypoxia were immunohistochemically stained in tumor sections to visualize their spatial relationship and to find a possible explanation for the absence of a correlation between the in vitro and in vivo expression of pAKT.

We observed that EGFR expression was predominantly present in tissue surrounding blood vessels, whereas pAKT was mostly expressed further away from the vessels in hypoxic areas indicated by pimonidazole staining in the consecutive tumor section (Figure 3 and Additional file 2: Figure S2). Using quantitative image analysis, we observed that in average only $7.7 \%$ (range: 1.0-33.6\%) of cells expressing EGFR also expressed pAKT. Thus, although cells were present that expressed both EGFR and pAKT, the overall overlap was relatively low. Moreover, we found that the fraction of the tumor positive for pimonidazole (hypoxic fraction) was significantly correlated with the fraction positive for pAKT $\left(r_{s}=0.51, p=0.04\right)$ (Figure 4$)$. These results suggest that pAKT is activated by hypoxia in an EGFR-independent way in these tumors.

pAKT is induced by hypoxia in vitro, and is important for cell survival under hypoxia

To confirm that hypoxia itself is an activating factor for AKT in vivo, cells of two HNSCC lines were cultured under hypoxic conditions $\left(\begin{array}{lll}0.5 \% & \mathrm{O}_{2}\end{array}\right)$. One hour after hypoxia, pAKT was indeed increased in both cell lines 

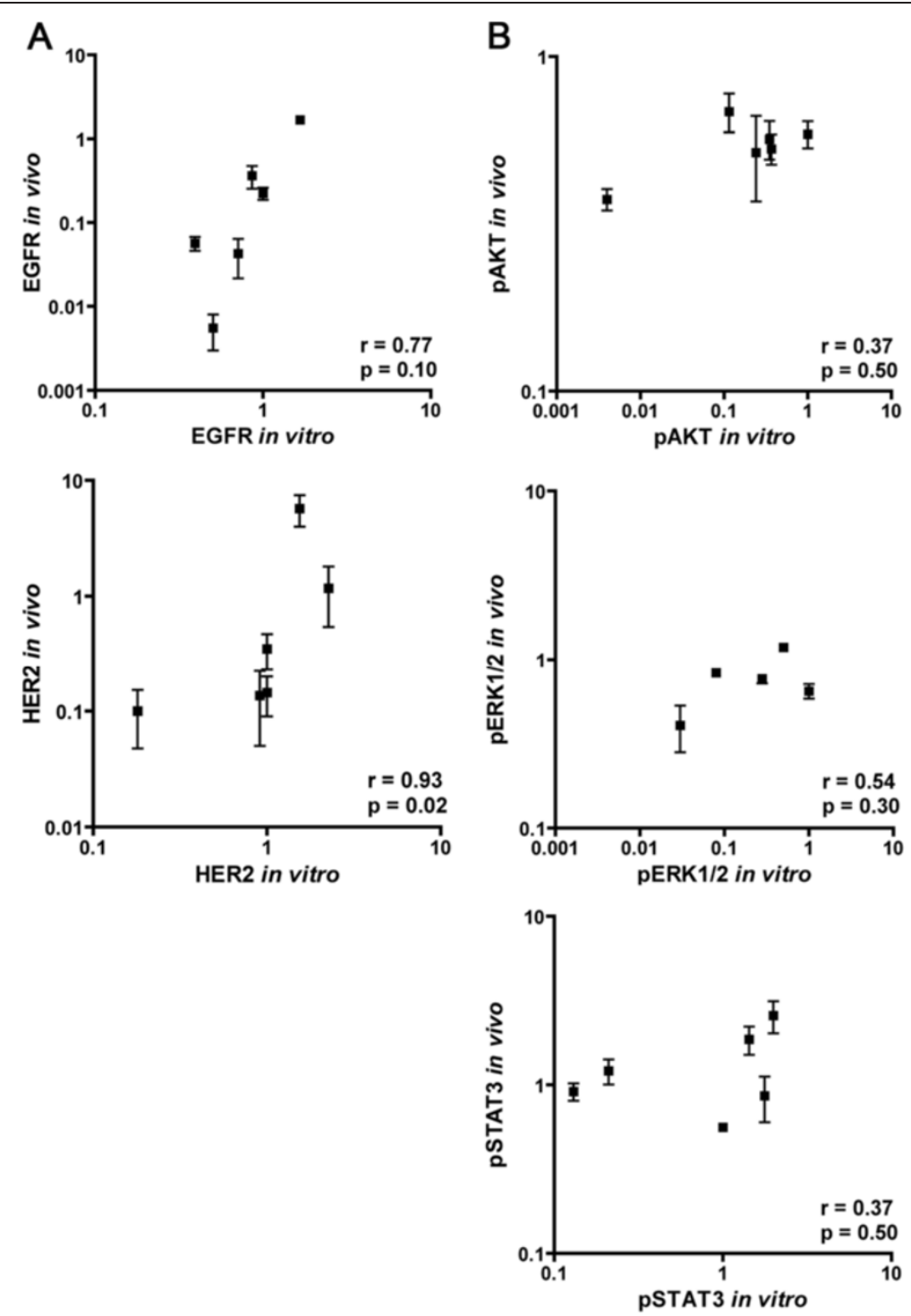

Figure 2 Correlation between in vitro and in vivo expression of EGFR/HER2 and pAKT/pSTAT3/pERK1/2 in 6 HNSCC lines. A) Correlation between in vitro and in vivo expression of EGFR and HER2. B) Correlation between in vitro and in vivo expression of pAKT, pERK1/2 and pSTAT3. Expression was assessed by western blot analysis and depicted in relative units. The integrated optical density (IOD) was measured and all values were normalized to those of a-tubulin by dividing the IOD value for that specific marker by the IOD value of a-tubulin. In vitro expression of UT-SCC5 was set as standard. Error bars represent standard error of the mean and all axes are in log scale. Correlations between in vitro and in vivo expression were assessed using the Spearman correlation test.

(Figure 5A). As pAKT plays an important role in cell survival, these results suggest that hypoxic cells could be specifically targeted by pAKT-inhibition. To test the hypothesis that hypoxic cells are more sensitive to pAKTinhibition, both normoxic and hypoxic $\left(0.5 \% \mathrm{O}_{2}\right)$ cells were treated with the specific pAKT-inhibitor MK-2206 and cell survival was assessed. MK-2206 treatment decreased pAKT expression efficiently under normoxic and hypoxic conditions (Figure 5A). In both HNSCC cell lines hypoxia itself did not cause a decrease in cell survival (Figure 5B). Under normoxic conditions, MK-2206 had a small, but non-significant, effect on cell survival.
However, when hypoxic cells were treated with MK2206 this resulted in a significant decrease in cell survival $(p<0.01)$. These results indicate that activation of AKT by hypoxia is important for cell survival under hypoxic conditions and can be specifically targeted by MK-2206.

\section{Discussion}

In recent years, a variety of newly developed targeted anticancer therapies have successfully been combined with classic therapies, including the combination of EGFRinhibition and radiotherapy in HNSCC [2]. However, these 


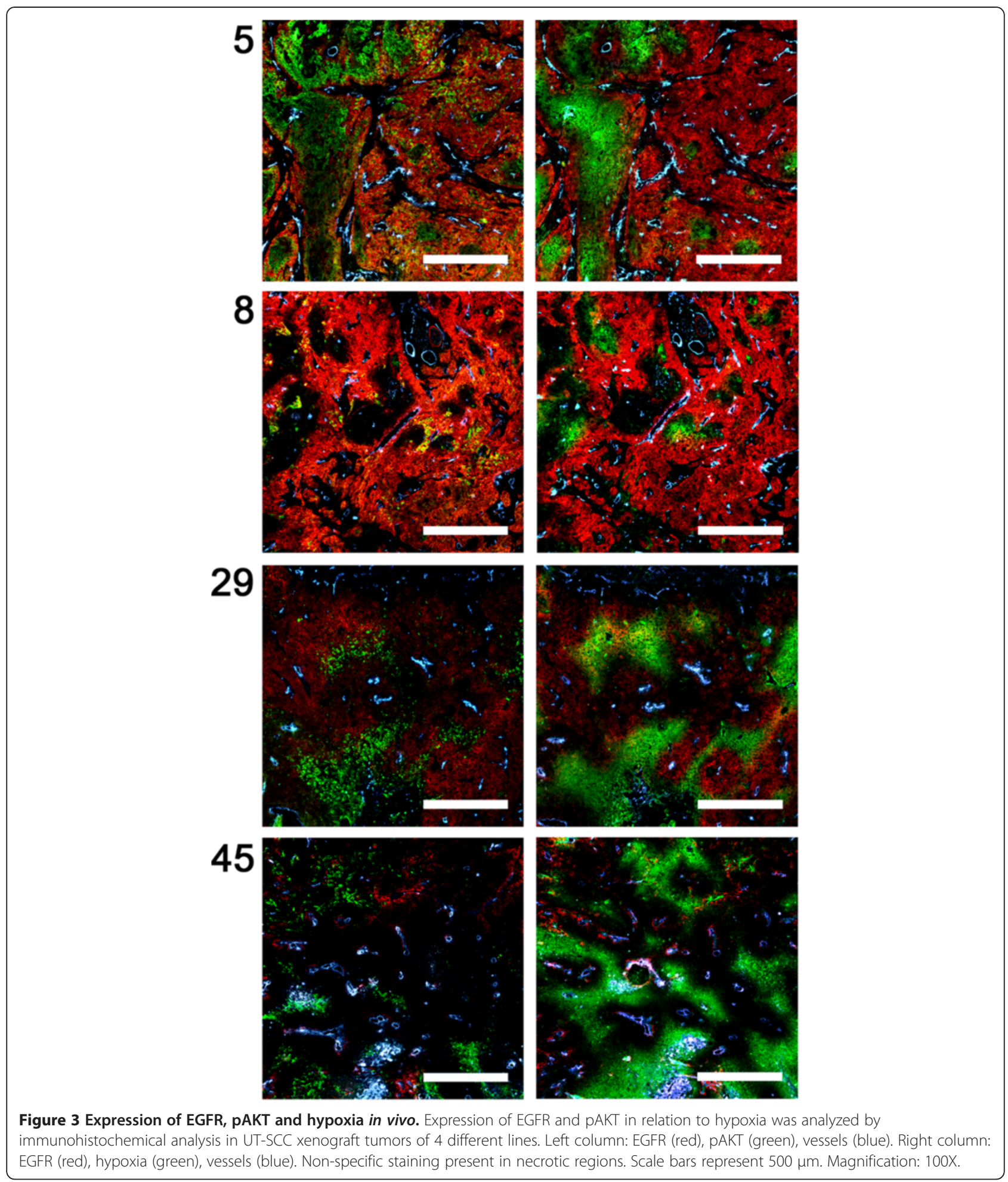

new intensified combinations also induce increased toxicity and (acquired) resistance $[3,13]$. The $a$ priori selection of patients that will respond is thus paramount, and research has focused on the identification of resistance mechanisms using different in vitro models. However, the complex tumor microenvironment can also be a source of therapy resistance. Hypoxia can have a great impact on the behavior of a tumor cell and also on the response to treatment [14]. Therefore, it is important to determine which proteins are influenced by hypoxia, because these 


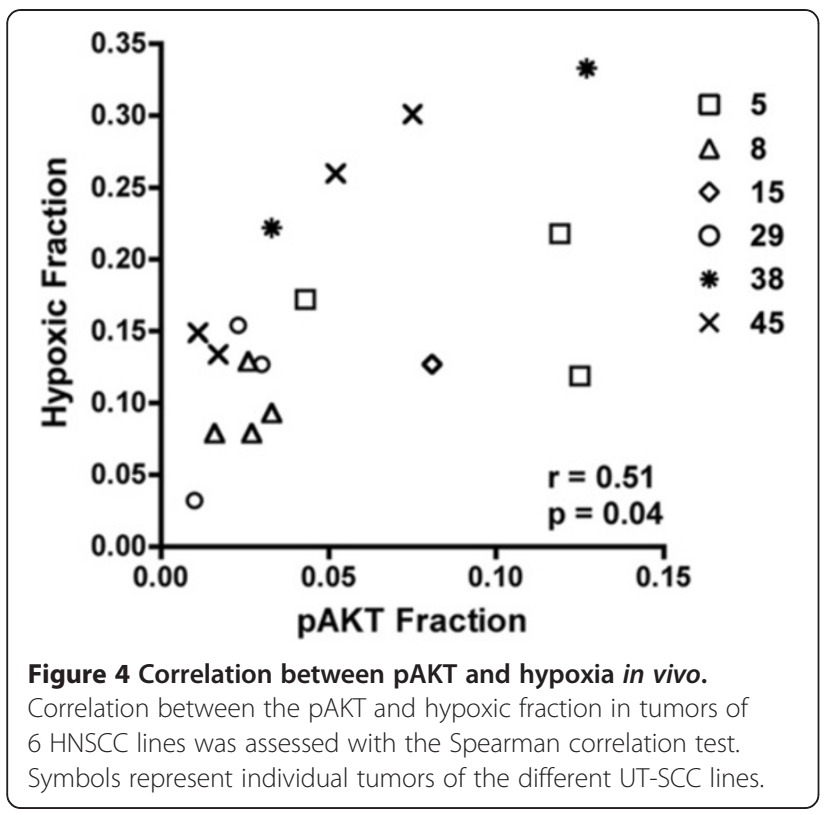

proteins could be potential targets to reduce therapy resistance to a variety of treatments, including radiotherapy and EGFR-inhibition.

In the current study, we found that the expression of EGFR and HER2 in vitro was correlated with the in vivo expression, which indicates that the expression of these tyrosine kinase receptors is largely an intrinsic feature of a tumor cell. The in vitro expression of activated EGFR (pEGFR) was also highly correlated with the in vivo expression, while the levels of pHER2 in vivo were clearly higher and varied more in the xenografts than in vitro. These observations suggest that activation of EGFR is possibly more genetically determined, e.g. by mutations present in the different tumor lines, while the activation of HER2 is more determined by factors in the tumor microenvironment that are not present in standard 2D cell culture. Also, in head and neck cancer patients a strong correlation between EGFR and pEGFR levels has been observed [15]. This correlation supports our observation that the extend of activated EGFR is predominantly influenced by EGFR overexpression and not by other stimuli in the microenvironment.

In contrast to the tyrosine kinase receptors, no in vitro-in vivo correlation was observed for the activated kinases pAKT, pERK1/2 or pSTAT3, indicating that the expression of these activated kinases is influenced by factors in the tumor microenvironment. Here, we show that hypoxia is an activating stimulus for AKT in vivo. This hypoxia-induced increase in pAKT cannot be explained by hypoxia-induced activation of EGFR as is observed in different in vitro models [16,17]. EGFR expression was namely predominantly present in oxygenated areas and there was a relatively low overlap between EGFR and pAKT expression in vivo. Besides these observations in our preclinical models, mismatch in EGFR-pAKT expression and the presence of pAKT in hypoxic regions is also observed in HNSCC patient samples [8]. EGFR-independent upregulation of pAKT by hypoxia has also been observed in lung cancer cells,

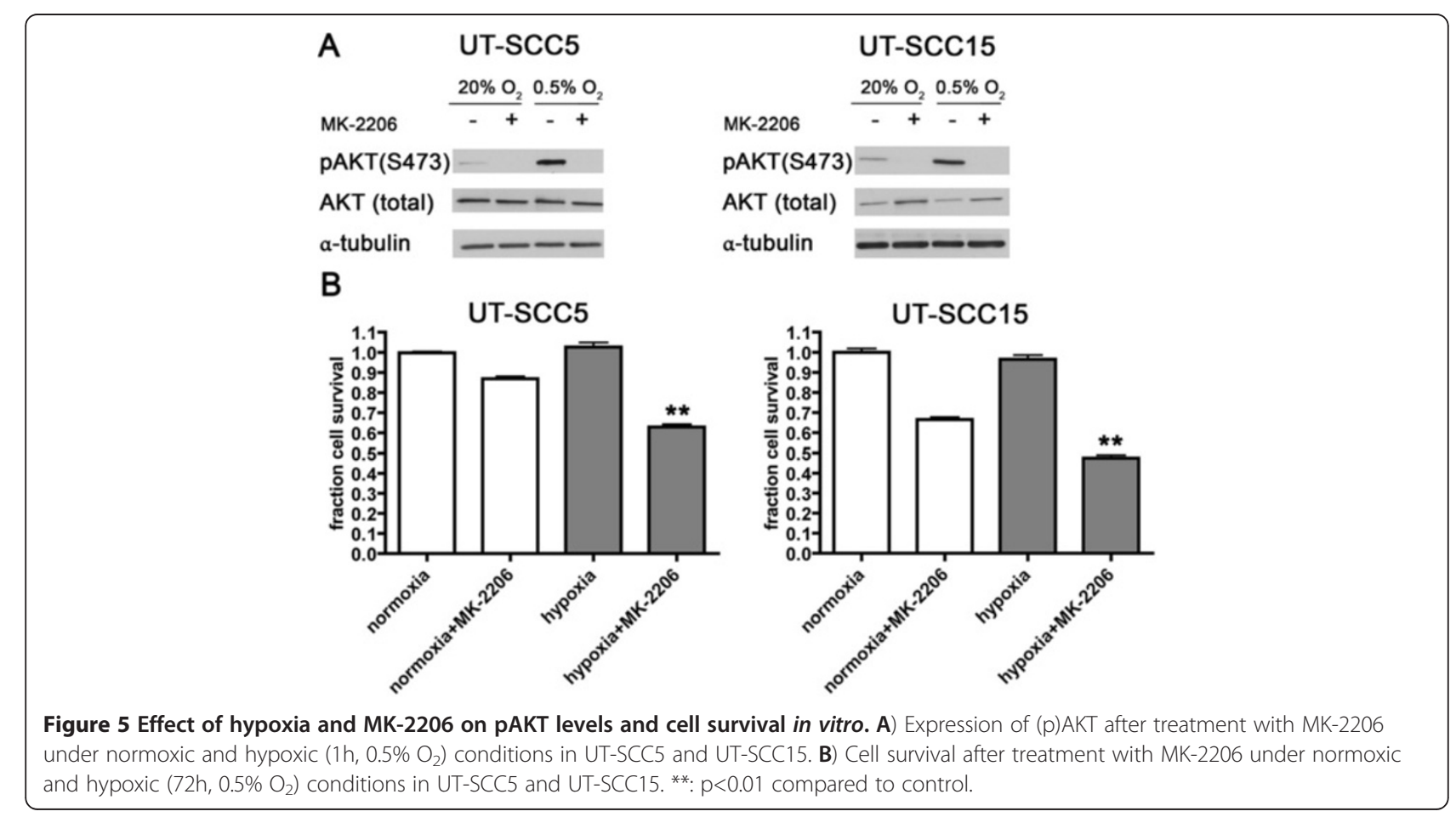


whereby activation of AKT was induced via the IGF1R/ PI3K/AKT pathway [18]. Also oxidative stress, which can occur during reoxygenation, has been shown to activate AKT in HNSCC cells [19]. Hypoxia-induced, EGFR-independent, activation of AKT could thus be an important resistance mechanism in HNSCC patients treated with EGFR-inhibition and radiotherapy. Although EGFR is the most commonly overexpressed tyrosine kinase receptor in head and neck cancer, also other receptors are overexpressed like HER2, HER3, and IL-6 receptor $[20,21]$, which could possibly play a role in hypoxia-induced activation of AKT. However, we focused on our major finding that activation of AKT is a characteristic of hypoxic cells in HNSCC and therefore a potential target to specifically kill hypoxic cells. Extensive crosstalk between different growth factor receptors, such as EGFR and MET, has been reported [22]. These growth factor receptors activate similar pathways, which means that cells that overexpress multiple growth factor receptors can sustain survival signaling even if one of the receptors is blocked [23]. This is exemplified by the study of Erjala et al., which also used a panel of UT-SCC cell lines, that showed that EGFR or pEGFR levels were not correlated, but pHER2 and HER3 levels were correlated with sensitivity to EGFR-inhibition [4]. Also downstream signaling molecules like pAKT en pERK1/2 were not correlated with sensitivity for EGFR-inhibition. In our cell lines, we did also not observe that overexpression of pEGFR was consistently linked to overexpression of pSTAT3, pAKT or pERK1/2. Therefore, it is more important to determine activation of the common downstream pathway, which is responsible for cell survival, as this will be a more attractive target to overcome treatment resistance than targeting one specific growth factor receptor. In the HNSCC tumor lines studied, we indeed show that pAKT-inhibition decreases cell survival in hypoxic cells, but not in normoxic cells. Hypoxic cells are resistant to a variety of treatment regimens, including radiotherapy [9], and as pAKT signaling is an important cell survival pathway [24], targeting of pAKT in hypoxic tumors could be a promising way to significantly improve patient outcome. Additionally, multiple animal studies have shown that MK-2206 also inhibits pAKT in vivo and reduces tumor growth [25-27]. Moreover, Knowles et al. showed that MK-2206 not only reduced primary tumor size in an orthotopic HNSCC model, but also inhibited HNSCC migration in vitro and reduced the number of lymph node metastases in vivo [25]. Although the effect of AKT-inhibition on HNSCC migration could explain the reduced metastases formation in this study, it is also known that hypoxia can induce a metastatic phenotype [28]. Killing hypoxic cells via pAKT-inhibition, as we show in this study, could thus potentially reduce not only tumor growth, but also the metastatic potential of the tumor. AKT is also a highly druggable target in the clinic since multiple specific AKT inhibitors, including the inhibitor we used in our study, are already tested in phase I/II clinical trials and are generally well tolerated [29].

Although our data show that the microenvironment can induce the expression of activated kinases and that therefore expression levels in tumors do not correspond with cells in vitro, they do not explain why we see very little variation in total expression between the tumors. A possible reason for this is the technique we used to determine expression levels. Western blot analysis determines the total expression in all tumor cells together. However, using immunohistochemistry we observed that the level of expression of the different proteins varied widely between cells in the tumors under the influence of e.g. hypoxia and this spatial information is totally lost by western blot analysis. Thus, possibly by determining the expression in all cells together, these differences between individual cells level out and result in an 'average' level of expression, which differs relatively little between tumors as we observed in this study. One of the main advantages of immunohistochemistry is the possibility to analyze specifically tumor cells. Although we used tumors that had a large fraction of viable tumor cells and a very low amount of stromal cells, we cannot exclude the possibility that the presence of small amounts of normal cells affected our results.

\section{Conclusion}

Our data indicate that the expression of (p)EGFR and HER2 is largely an intrinsic feature of a tumor cell, while the in vivo expression of activated kinases of important cell signaling pathways can be substantially affected by the tumor microenvironment. Moreover, we show that AKT is activated by hypoxia both in vivo and in vitro and that hypoxic cells are more sensitive to AKT-inhibition. As hypoxic tumors are resistant to a variety of treatment regimens, AKT might thus be a promising druggable target in hypoxic tumors. Further research is warranted to confirm our hypothesis that anti-cancer treatment can be improved by specifically targeting hypoxic cells with pAKT-inhibition and in which way this finding can be optimally used to improve patient outcome in the future.

\section{Additional files} Additional file 1: Figure S1. In vitro and in vivo expression of (p)HER2,
(p)STAT3 and (p)ERK1/2 in 6 HNSCC lines. Cell lines were both cultured as
cell lines (in vitro) and grown as xenograft tumors (in vivo) and expression
levels were determined with western blot. A) In vitro expression of (p)
HER2, (p)STAT2 and (p)ERK1/2. B) In vivo expression of (p)HER2, (p)STAT2
and (p)ERK1/2. Number of harvested tumors ranged from 2 to 4 per cell
line. 
Additional file 2: Figure S2. Enlarged detail of Figure 3. Expression of EGFR, pAKT and hypoxia in a tumor of UT-SCC5. Left picture: EGFR (red), pAKT (green), vessels (blue). Right picture: EGFR (red), hypoxia (green), vessels (blue). Magnification: 100X.

\section{Competing interests}

The authors declare that they have no competing interests.

\section{Authors' contributions}

HS designed and coordinated the project, performed the animal experiments and western blot analyses and drafted the manuscript. JHK, AJK, and JB obtained funding for this project and participated in its design and coordination and drafted the manuscript. PNS helped with the statistical analyses and interpretation of the data and revised the manuscript. DLW and Ml participated in the design and interpretation of the data. SJW and MMV designed and performed the cell culture experiments. RG provided the cell lines and revised the manuscript. All authors read and approved the final manuscript.

\section{Acknowledgements}

We are grateful to Mr. P. Rijken and Mr. J. Lok for their valuable input and excellent technical assistance. This project was financially supported by the Dutch Cancer Society (grant number 2008-4000) and, in part by the Clinical and Translational Science Award (CTSA) program, through the NIH National Center for Advancing Translational Sciences (NCATS), grant UL1TR000427 (DLW). The content is solely the responsibility of the authors and does not necessarily represent the official views of the $\mathrm{NIH}$

\section{Author details}

'Department of Radiation Oncology, Radboud University Nijmegen Medical Centre, PO Box 9101, 6500 HB, Nijmegen, The Netherlands. '²epartment of Human Oncology, University of Wisconsin School of Medicine and Public Health, 1111 Highland Ave, Madison WI 53705, USA. ${ }^{3}$ Department of Otorhinolaryngology-Head and Neck Surgery and Department of Medical Biochemistry, Turku University Hospital and University of Turku, PO Box 52, Fl-20521, Turku, Finland.

Received: 9 July 2012 Accepted: 5 October 2012

Published: 10 October 2012

\section{References}

1. Davies L, Welch HG: Epidemiology of head and neck cancer in the United States. Otolaryngol Head Neck Surg 2006, 135(3):451-457.

2. Bonner JA, Harari PM, Giralt J, Azarnia N, Shin DM, Cohen RB, Jones CU, Sur R, Raben $D$, Jassem J, et al: Radiotherapy plus cetuximab for squamous-cell carcinoma of the head and neck. N Engl J Med 2006, 354(6):567-578.

3. Wheeler DL, Dunn EF, Harari PM: Understanding resistance to EGFR inhibitors-impact on future treatment strategies. Nat Rev Clin Oncol 2010, 7(9):493-507.

4. Erjala K, Sundvall M, Junttila T, Zhang N, Savisalo M, Mali P, Kulmala J, Pulkkinen J, Grenman R, Elenius K: Signaling via ErbB2 and ErbB3 associates with resistance and epidermal growth factor receptor (EGFR) amplification with sensitivity to EGFR inhibitor gefitinib in head and neck squamous cell carcinoma cells. Clin Cancer Res 2006, 12(13):4103-4111.

5. Yamatodani T, Ekblad L, Kjellen E, Johnsson A, Mineta H, Wennerberg J: Epidermal growth factor receptor status and persistent activation of Akt and p44/42 MAPK pathways correlate with the effect of cetuximab in head and neck and colon cancer cell lines. J Cancer Res Clin Oncol 2009, 135(3):395-402.

6. Bonner JA, Yang ES, Trummell HQ, Nowsheen S, Willey CD, Raisch KP: Inhibition of STAT-3 results in greater cetuximab sensitivity in head and neck squamous cell carcinoma. Radiother Oncol 2011, 99(3):339-343.

7. Song Jl, Grandis JR: STAT signaling in head and neck cancer. Oncogene 2000, 19(21):2489-2495.

8. Bussink J, van der Kogel AJ, Kaanders JH: Activation of the PI3-K/AKT pathway and implications for radioresistance mechanisms in head and neck cancer. Lancet Oncol 2008, 9(3):288-296.

9. Janssens $\mathrm{GO}$, Rademakers $\mathrm{SE}$, Terhaard $\mathrm{CH}$, Doornaert PA, Bijl HP, van den Ende P, Chin A, Marres HA, de Bree R, van der Kogel AJ, et al: Accelerated
Radiotherapy With Carbogen and Nicotinamide for Laryngeal Cancer: Results of a Phase III Randomized Trial. J Clin Oncol 2012, 30(15):1777-1783.

10. Overgaard J: Hypoxic radiosensitization: adored and ignored. J Clin Oncol 2007, 25(26):4066-4074.

11. Rademakers SE, Span PN, Kaanders JH, Sweep CGJ, van der Kogel AJ, Bussink J: Molecular aspects of tumour hypoxia. Mol Oncol 2008, 2:41-53.

12. Ratushny V, Astsaturov I, Burtness BA, Golemis EA, Silverman JS: Targeting EGFR resistance networks in head and neck cancer. Cell Signal 2009, 21(8):1255-1268.

13. Giro C, Berger B, Bolke E, Ciernik IF, Duprez F, Locati L, Maillard S, Ozsahin $M$, Pfeffer R, Robertson AG, et al: High rate of severe radiation dermatitis during radiation therapy with concurrent cetuximab in head and neck cancer: results of a survey in EORTC institutes. Radiother Oncol 2009, 90(2):166-171.

14. Harris AL: Hypoxia-a key regulatory factor in tumour growth. Nat Rev Cancer 2002, 2(1):38-47.

15. Thariat J, Etienne-Grimaldi MC, Grall D, Bensadoun RJ, Cayre A, Penault-Llorca F, Veracini L, Francoual M, Formento JL, Dassonville O, et al: Epidermal growth factor receptor protein detection in head and neck cancer patients: a many-faceted picture. Clin Cancer Res 2012, 18(5):1313-1322

16. Franovic A, Gunaratnam L, Smith K, Robert I, Patten D, Lee S: Translational up-regulation of the EGFR by tumor hypoxia provides a nonmutational explanation for its overexpression in human cancer. Proc Natl Acad Sci U S A 2007, 104(32):13092-13097.

17. Wang X, Schneider A: HIF-2 \{alpha\}-mediated activation of the epidermal growth factor receptor potentiates head and neck cancer cell migration in response to hypoxia. Carcinogenesis 2010, 31(7):1202-1210.

18. Kim TR, Cho EW, Paik SG, Kim IG: Hypoxia-induced SM22alpha in A549 cells activates the IGF1R/PI3K/Akt pathway, conferring cellular resistance against chemo- and radiation therapy. FEBS Lett 2012, 586(4):303-309.

19. Leopoldino AM, Squarize CH, Garcia CB, Almeida LO, Pestana CR, Sobral LM, Uyemura SA, Tajara EH, Silvio Gutkind J, Curti C: SET protein accumulates in HNSCC and contributes to cell survival: Antioxidant defense, Akt phosphorylation and AVOs acidification. Oral Oncol, In press.

20. Wei Q, Sheng L, Shui Y, Hu Q, Nordgren H, Carlsson J: EGFR, HER2, and HER3 expression in laryngeal primary tumors and corresponding metastases. Ann Surg Oncol 2008, 15(4):1193-1201.

21. Chen CC, Chen WC, Lu CH, Wang WH, Lin PY, Lee KD, Chen MF: Significance of interleukin-6 signaling in the resistance of pharyngeal cancer to irradiation and the epidermal growth factor receptor inhibitor. Int J Radiat Oncol Biol Phys 2010, 76(4):1214-1224.

22. Guo A, Villen J, Kornhauser J, Lee KA, Stokes MP, Rikova K, Possemato A Nardone J, Innocenti G, Wetzel R, et al: Signaling networks assembled by oncogenic EGFR and c-Met. Proc Natl Acad Sci U S A 2008, 105(2):692-697.

23. Wheeler DL, Huang S, Kruser TJ, Nechrebecki MM, Armstrong EA, Benavente $S$, Gondi V, Hsu KT, Harari PM: Mechanisms of acquired resistance to cetuximab: role of HER (ErbB) family members. Oncogene 2008, 27(28):3944-3956.

24. Vivanco I, Sawyers CL: The phosphatidylinositol 3-Kinase AKT pathway in human cancer. Nat Rev Cancer 2002, 2(7):489-501.

25. Knowles JA, Golden B, Yan L, Carroll WR, Helman EE, Rosenthal EL: Disruption of the AKT pathway inhibits metastasis in an orthotopic model of head and neck squamous cell carcinoma. Laryngoscope 2011, 121(11):2359-2365

26. Meng J, Dai B, Fang B, Bekele BN, Bornmann WG, Sun D, Peng Z, Herbst RS, Papadimitrakopoulou V, Minna JD, et al: Combination treatment with MEK and AKT inhibitors is more effective than each drug alone in human non-small cell lung cancer in vitro and in vivo. PLoS One 2010, 5(11):e14124.

27. Sangai T, Akcakanat A, Chen H, Tarco E, Wu Y, Do KA, Miller TW, Arteaga CL, Mills GB, Gonzalez-Angulo AM, et al: Biomarkers of Response to Akt Inhibitor MK-2206 in Breast Cancer. Clin Cancer Res, In press.

28. Bristow RG, Hill RP: Hypoxia and metabolism. Hypoxia, DNA repair and genetic instability. Nat Rev Cancer 2008, 8(3):180-192.

29. Andersson T, Alfredsson L, Kallberg H, Zdravkovic S, Ahlbom A: Calculating measures of biological interaction. Eur J Epidemiol 2005, 20(7):575-579.

doi:10.1186/1471-2407-12-463

Cite this article as: Stegeman et al: Activation of AKT by hypoxia: a potential target for hypoxic tumors of the head and neck. BMC Cancer 2012 12:463. 